\title{
»Nicht nur religiös, sondern auch politisch»
}

\author{
Über eine unverkrampfte Dreiecksbeziehung von geistli- \\ chem, kirchlichem und politischem Amt
}

Esther Straub

\begin{abstract}
"In der Voraussetzung, dass ein Professor der Theologie nicht verpflichtet sei, diejenigen politischen und sozialen Ansichten zu vertreten, die in einem bestimmten Jahrzehnt zufällig gerade herrschend sind, fühle ich mich mit den meinigen innerhalb der theologischen Fakultät wie der Universität überhaupt völlig an meinem Platze und habe ein gutes Gewissen dabei. Ja es fällt mir gerade um dieser Überzeugungen willen schwer, meine akademische Stellung aufzugeben, da leicht der Schein erregt werden könnte, als sei meine Demission ein Zugeständnis an jene, die mir das moralische Recht zu einer Wirksamkeit an der Universität deswegen bestreiten, weil ich nicht nur religiös sondern auch politisch, sozial und pädagogisch anders als sie denke und weil ich mir das Recht genommen habe, mein religiöses Bekenntnis auch ins politische, soziale und kulturelle Leben hineinzutragen, statt es sorgfältig in eine nur Fachleuten bekannte Theologie einzuschließen und damit unwirksam zu machen. «1
\end{abstract}

Mit diesen Worten demissionierte Leonhard Ragaz als Professor an der Universität Zürich und kehrte der theologischen Fakultät den Rücken. In seinem Schreiben an den Regierungsrat hielt er fest, es sei kein Rückzug vor dem akademischen Geist, den er antrete, sondern es sei vor allem seine Stellung zur heutigen Kirche, die ihm seine jetzige Arbeit unmöglich gemacht habe. Als Professor der systematischen Theologie hatte Ragaz im Nebenamt auch die praktische zu lehren und Pfarrer in den Kirchendienst einzuführen. Er sei immer mehr im Glauben erschüttert worden, dass die heutige Kirche noch ein Gefäss der Wahrheit Gottes sein könne. Dieselben religiösen Überzeugungen, die seine Stellung zur Kirche begründeten, hätten ihn jedoch auch in Konflikt mit den Formen des heutigen Fakultätsbetriebs gebracht: »Ich müßte, um in voller Wahr-

\footnotetext{
1 Aus dem Rücktrittsschreiben von Leonhard Ragaz an die Erziehungsdirektion des Kantons Zürich, datiert vom 24. Mai 1921. Das Schreiben findet sich im Artikel von Leonhard Ragaz, Warum ich meine Professur aufgegeben habe?, in: Neue Wege 15/7-8 (1921), 283-293, hier 284.
} 
heit, das heißt: in genügender Übereinstimmung zwischen meinem Verständnis der Sache Christi und meiner Art, diese zu vertreten, weiter an der Fakultät wirken zu können, eine Umgestaltung des ganzen theologischen Lehrsystems voraussetzen, die für nahe Zeiten zu erhoffen, ich nicht mehr den Mut habe. $\mathbb{1}^{2}$ Nicht die Kenntnis der Bibel und der Geschichte sei ihm ein Problem gewesen, sondern das religiöse Gedankensystem, das an Stelle des unmittelbaren Tuns und Erlebens getreten sei.»Ich glaube, daß dies eine der schwersten Gefahren ist, die unser Christentum überhaupt belasten, hatte aber immer das Gefühl, daß es besonders bedenklich sei,junge Menschen in ein solches zu stecken, ja, daß dies das sicherste Mittel sei, sie von dem wirklichen Gotte und seinem Reiche abzulenken. $\mathbb{3}^{3}$

In seiner Überzeugung habe er den Schnitt machen müssen. "Sollte ich geirrt haben, so will ich lieber in voller Wahrhaftigkeit einen falschen Weg gehen - falls dies möglich sein sollte - als vor mir selbst mit dem Verdacht belastet sein, das Letzte und Schwerste aus Mangel an Kraft und Glauben nicht gewagt zu haben. $\aleph^{4}$

Fast hundert Jahre alt ist sie, diese Episode aus der Geschichte der Zürcher theologischen Fakultät. Aus Resignation über die verbürgerlichte Kirche hatte Leonhard Ragaz damals den Lehrbetrieb verlassen, um sich ganz der religiös-sozialen Arbeit für Pazifismus und Weltfrieden und der Arbeiterbildung zu widmen.Vierzehn Jahre später, 1935, trat er nach 23 Jahren Mitgliedschaft aus der Sozialdemokratischen Partei aus, weil sie den Antimilitarismus aufgegeben hatte. Auch darin versuchte Ragaz wahrhaftig zu sein.

Theologie, Kirche und Politik: Befindet sich in einem Bermudadreieck, wer politisches Engagement aus theologischer Motivation betreibt und Kirche mit ihm verbindet? Oder konkret: Kann ein und dieselbe Person drei Ämter als Pfarrerin, Kirchenrätin und Kantonsrätin ausüben? ${ }^{5}$ Die Antwort auf die Anfrage zu diesem Essay soll eine biographische sein und Einblick geben, mit welchen Notwendigkeiten und welchen Vorbehalten die Arbeit in Kirche und Politik konfrontiert ist. Sie orientiert sich an den drei Seiten des Dreiecks von Pfarramt, kirchlichem Leitungsamt und politischem Amt.

2 Ebd., 285.

Ebd., 288.

Ebd., 289.

5 So lautete die Anfrage zu diesem Essay: "Da Sie sowohl Kantonsrätin als auch Kirchenrätin sind, wären wir sehr daran interessiert, wenn Sie Ihre Rolle reflektieren würden.» 


\section{Die Hypotenuse des Dreiecks - oder: Die Bezie- hung zwischen Pfarramt und Politik}

Als ich Ende 2002 die theologische Fakultät verliess, geschah dies weniger aus Ärger über die Kirche als aus dem Willen heraus, theologische Wahrheiten in der kirchlichen Praxis zu erproben. Bereits einige Jahre zuvor war ich in die Sozialdemokratische Partei eingetreten und engagierte mich mittlerweile als Präsidentin meiner Sektion. In der Kirchgemeinde Zürich Schwamendingen fand ich meine eigentliche Traumpfarrstelle in einem Stadtquartier, das seine ländliche Herkunft mit einem regen Vereinsleben genauso stolz zur Schau trägt wie seine Multikulturalität, die es als typisches Arbeiterquartier prägt. Die Parteistärken von SP und SVP (leicht schwächer) halten sich in Schwamendingen seit zwanzig Jahren fast die Waage, während der Stadtkreis früher eine Hochburg der SP war. Die Kirchgemeinde weiss denn auch um eine lange Tradition religiös-sozialer Pfarrpersonen von Adolf Maurer über Matthias Thurneysen bis zu Ueli Hedinger - und seit 2008 ist der Kirchturm der Alten Kirche St. Niklaus (allerdings aufgrund eines Irrtums der Denkmalpflege) gar in leuchtendem Rot gestrichen.

Knapp drei Jahre nach meinem Pfarramtsantritt wurde ich als Vertreterin des Stadtkreises 6 in den Gemeinderat der Stadt Zürich gewählt. Niemand hustete in der Kirchgemeinde, keiner muckte auf. Weiterhin besuchten SVP-Wähler und SP-Wählerinnen gleichermassen die Gottesdienste. Der Umstand, dass ich mit ersteren oft einen besonders guten Kontakt pflege, brachte mir bei meinem Pfarrkollegen gar den Ruf ein, eine "SVP-Pfarrerin" zu sein. Predigten mit politischer Aussagekraft ${ }^{6}$ werden in der Kirchgemeinde geschätzt, unterschiedliche Meinungen beim sonntäglichen Kirchenkaffee oder bei anderer Gelegenheit ausdiskutiert. Tatsächlich: Wahrhaftigkeit wird in dieser Kirchgemeinde grossgeschrieben. Die Gemeindeglieder haken meine aktive Parteiarbeit als mein persönliches politisches Engagement ab und thematisieren es kaum. Mag sein, dass kritische Stimmen den Weg nicht bis zu mir finden, Ablehnung erlebte ich jedenfalls nur ein einziges Mal, als ein auswärtiger Besucher sich aufgrund einer feministischen Aussage brüskiert fühlte.

\footnotetext{
${ }^{6}$ Selbstverständlich orientiert sich eine Predigt nicht am Parteiprogramm, sondern an den biblischen Schriften und ihrem Kerygma. Ganz nach der Weisheit von Ernst Käsemann: "Wenn Christen sich für Humanismus, Kultur und eine bessere Politik einsetzen, tun sie das von jener durch das Kreuz markierten Grenze her, auf der es den Dienst nicht ohne das Ärgernis gibt. "Ernst Käsemann, Der weite Weg, in: Ders., Der Ruf der Freiheit, Tübingen ${ }^{51972,186-224, ~ h i e r ~} 195$.
} 
Umgekehrt rückt mein Beruf im Amt als Gemeinde- bzw. seit drei Jahren als Kantonsrätin oft in den Vordergrund. Nicht nur werde ich von Ratskollegen oder -kolleginnen für Kasualfeiern angefragt, auch wechselt das Gespräch untereinander schnell einmal von der politischen auf eine theologische Inhaltsebene und zurück. Während Apothekerinnen, Bauern, Lehrpersonen oder Gewerbetreibende selbstverständlich im Parlament vertreten sind und Anliegen aus ihrem Berufsfeld ins Gespräch bringen, tut die Kirche gut daran, dafür zu sorgen, dass sie nicht aussen vor bleibt. Als Pfarrerin leiste ich im Parlament nebenher auch Öffentlichkeitsarbeit für die Kirche.

\section{Die Ankathete - oder: Die Schwierigkeit, als Pfar- rerin in den Kirchenrat gewählt zu werden}

Vor zwei Jahren wurde ich von der Synode der Zürcher Landeskirche als Vertreterin der Religiös-sozialen Fraktion in den Kirchenrat gewählt. Meine Wahl in die Exekutive war umstritten, und die Präsidien der drei anderen Fraktionen versuchten, sie mit einer früh lancierten Gegenkandidatur zu verhindern. Als Grund, weshalb ich nicht wählbar sei, führten sie an, dass mit mir eine Mehrheit von Pfarrpersonen im Kirchenrat Einsitz nähme, was zwar mit der Kirchenordnung vereinbar wäre, aber einer säkularen Gesellschaft nicht gerecht würde. ${ }^{7}$ Dass eine presbyterial-synodale Grundstruktur der Kirche das Leitungsamt nicht auf ordinierte Kirchenglieder eingrenzt, hat sich in der reformierten Geschichte zweifellos bewährt. Es ist jedoch keineswegs zwingend, die ordinierten Glieder in die Minderheit zu setzen. Und gegenüber der Auffassung, einer Kirchenleitung stehe im Kontext der säkularen Gesellschaft etwas weniger Theologie gut an, gilt es zu bedenken, dass es insbesondere die Theologie ist, die die Übersetzungsarbeit zwischen Kirche und säkularer Gesellschaft leistet.

Was die Kritikerinnen und Kritiker einer theologischen Mehrheit im Kirchenrat nicht thematisierten: Dass in der ganzen Geschichte der Zürcher Landeskirche erst ein einziges Mal eine Frau und Pfarrerin in die Exekutive gewählt worden war. Das gesamtgesellschaftliche Problem, dass Frauen in Leitungsgremien massiv untervertreten

7 So der Synodale Peter Schmid von der Evangelisch-kirchlichen Fraktion in der NZZ: "In einer säkularen Gesellschaft sollte im Kirchenrat nicht die Mehrheit Pfarrpersonen sein«. (Zitiert nach: Matthias Herren, Politiker übernehmen im Kirchenrat, in: Neue Zürcher Zeitung, 25.06.2015, https://www.nzz.ch/zuerich/politikeruebernehmen-im-kirchenrat-1.18568571 [31.10.2017]). 
sind, beschäftigt in Zürich die theologische Fakultät genauso wie die Kirche. Die Gleichstellung der Geschlechter, ein religiös-soziales Grundanliegen, für das sich vor hundert Jahren insbesondere Clara Ragaz stark gemacht hat, ${ }^{8}$ ist noch immer nicht Wirklichkeit geworden, auch wenn die reformierte Kirche im Unterschied zur katholischen Schwesterkirche Frauen heute immerhin auf dem Papier nicht mehr grundsätzlich diskriminiert.

\section{Die Gegenkathete - oder: Die Verbindung von Kirchenleitungsamt und politischem Amt}

Dass im Vorfeld der Kirchenratswahl die drohende TheologenMehrheit zu reden gab, wunderte die Neue Zürcher Zeitung. Im Gegenzug problematisierte sie unter dem Titel »Politiker übernehmen im Kirchenrat «" den Umstand, dass mit der FDP-Kantonsrätin Katharina Kull und mir gleich zwei Kantonsrätinnen in den Kirchenrat gewählt würden. Alfred Heer, der damalige Kantonalparteipräsident der SVP, von der ihrerseits bereits mehrmals Parteimitglieder im Kirchenrat vertreten waren, liess sich mit dem Satz zitieren: "Für mich ist diese Tendenz ein Schwächezeichen der Kirche « ${ }^{10}$. Die Kirche müsse aufgrund der Verkündigung des Evangeliums stark sein und nicht wegen ihrer "Verfilzung mit der Politik «"

Gerade als Glaubensgemeinschaft, die aus dem Evangelium lebt, ist die Kirche auf eine partizipative und demokratische Struktur ihrer Körperschaft ausgerichtet. ${ }^{12}$ Politische Erfahrung und ebensolches Know-how können in den Reihen ihrer Leitungsgremien nur ein Gewinn sein. Abgesehen davon ist die vom staatlichen Gefüge weitgehend entflochtene Zürcher Kirche heute tatsächlich auf eine gute Vernetzung mit Behörden und öffentlichen Institutionen angewiesen. Ein aufgeschlossener Kontakt fördert nicht nur ihr eigenes Wohl, auch der Staat profitiert vom Dienst der Kirche und von ihrer kritischen Begleitung ${ }^{13}$. Beispiele sind die Seelsorge in Institutionen

\footnotetext{
8 Vgl. z.B. Clara Ragaz, Die Revolution der Frau, in: Neue Wege 13/7 (1919), 361372.

9 Herren, Politiker übernehmen im Kirchenrat.

${ }^{10}$ Ebd.

${ }^{11}$ Ebd.

12 Vgl. Esther Straub, Strukturreform und Pneuma. Warum die fast schon absolute Minderheitsposition der Kirche selig macht, in: Neue Wege 111/6 (2017), 27-29.

${ }^{13}$ Vgl. das "prophetische Wächteramt" in Art. 4 Abs. 2 der Kirchenordnung der Evangelisch-reformierten Landeskirche des Kantons Zürich.
} 
wie Spitälern, Gefängnissen oder Asylzentren, ${ }^{14}$ das Mitwirken in der Härtefallkommission oder die Zusammenarbeit mit anderen Religionsgemeinschaften zur Förderung ihrer Integration. Als $\mathrm{Pa}-$ radebeispiel einer fruchtbaren engen Zusammenarbeit von Kirche und Staat sei die Gefängnisseelsorgekommission erwähnt, in der seit über 30 Jahren Vertretende des Amts für Justizvollzug, des Kirchenrats und Synodalrats, Gefängnisleitende, leitende Seelsorgende von christlicher und muslimischer Seite sowie Delegierte des psychiatrischen Dienstes, des Obergerichts, der Staatsanwaltschaft und der Entlassenenfürsorge miteinander im Gespräch sind, Problemlösungen suchen und in konkrete Schritte münden lassen.

Der Gefahr einer Verfilzung von Staat und Kirche wirken Transparenz und Wahrhaftigkeit in der Zusammenarbeit entgegen. Die reformierte Kirche ist aufgrund ihrer demokratischen, presbyterialsynodalenVerfassung eine verlässliche Partnerin des Staates. Auch die katholische Schwesterkirche ist als demokratische Körperschaft in Zürich seit über 50 Jahren vom Staat öffentlich-rechtlich anerkannt und arbeitet mit diesem in erfolgreicher Partnerschaft zusammen. Allerdings muss sie ihre demokratische Struktur in einem dualen System mit der hierarchischen und frauendiskriminierenden Struktur nach kanonischem Recht verbinden. Die Kontakte und erst recht die Geldflüsse zwischen den öffentlich-rechtlich anerkannten Kirchen und dem Staat sind transparent gestaltet. So zeigt zum Beispiel die von den Kirchen und dem Kanton bei der Universität Zürich in Auftrag gegebene Studie Kirchliche Tätigkeiten mit gesamtgesellschaftlicher Bedeutung im Kanton Zürich ${ }^{15}$ auf, dass die staatlichen Kostenbeiträge an die Kirchen gerechtfertigt sind.

\footnotetext{
${ }^{14}$ Vgl. Christoph Morgenthaler,Volkskirche - bescheiden, frech. Praktisch-theologische Thesen zur Zukunft der Volkskirche, in: Claudia Kohli Reichenbach, Matthias Krieg (Hg.), Volkskirche und Kirchenvolk. Ein Zwischenhalt, Zürich 2015, 69-75, hier 71: Zukunftsfähig sei "eine Kirche, die sich die Voraussetzungen bewahrt, im Spital oder im Gefängnis oder auf der Unfallstelle präsent bleiben zu dürfen, weil ihre Mitarbeiterinnen und Mitarbeiter dort professionell einen Beitrag leisten können, den andere so nicht leisten können."

15 Thomas Widmer, Kathrin Frey, Heiri Gander, Roman Zwicky, Pascale Münch, Kirchliche Tätigkeiten mit gesamtgesellschaftlicher Bedeutung im Kanton Zürich. Schlussbericht, Zürcher Politik- \& Evaluationsstudien Nr. 18, Zürich 2017, https:// www.ipz.uzh.ch/de/forschung/publikationen/publikationsreihen/ZuerchpolEva. html (31.10.2017).
} 


\section{Wahrhaftigkeit in der Zusammenarbeit von Kirche und Staat}

Der Umstand, dass ich als Pfarrerin, Kirchenrätin und Kantonsrätin jeweils beide Eckpunkte einer Dreiecksseite von innen kenne, sie repräsentiere und je nach Situation auch auseinanderhalten muss, ist nicht aussergewöhnlich. ${ }^{16}$ Dass die personelle Verknüpfung für alle Seiten förderlich ist, meine ich mit den kurzen Einblicken gezeigt zu haben. Mich persönlich leitet an den unterschiedlichen Orten ein Wort von Paulus aus seinem zweiten Brief an die Gemeinde in Korinth: "Wir haben aber dieselbe Geistkraft des Glaubens, von der geschrieben steht: Ich glaube, darum rede ich. So glauben auch wir, und darum reden wir" (2 Kor 4,13). Wahrhaftigkeit ist im Umgang, den Religion und Politik miteinander pflegen, ganz generell eine gute Ratgeberin. In einer Zeit, in der Religion schnell unter Verdacht gerät, im Staat ideologisch Einfluss nehmen zu wollen, und politische Verantwortungsträgerinnen und -träger in ihrem Umgang mit Religionsgemeinschaften verunsichert sind, tun sachliche und unverkrampfte Debatten not.

- Esther Straub promovierte an der Theologischen Fakultät der Universität Zürich. Sie ist Pfarrerin in Zürich Schwamendingen/Saatlen, Kirchenrätin der Evangelischreformierten Landeskirche des Kantons Zürich und politisiert für die SP im Zürcher Kantonsrat. Sie ist ausserdem Co-Präsidentin der 112-jährigen Zeitschrift Neue Wege.

${ }^{16} \mathrm{Vgl}$. z.B. die vielfältigen Interessenbindungen von Kantonsratsmitgliedern, die auf der Homepage des Kantonsrats offengelegt sind: http://www.kantonsrat.zh.ch/mitglieder/mitglieder.aspx (14.11.2017). 